\title{
On the Limits of Positioning-based Pedestrian Risk Awareness
}

\author{
Shubham Jain ${ }^{\dagger}$, Carlo Borgiattino ${ }^{\S}$, Yanzhi Ren ${ }^{\sharp}$, Marco Gruteser ${ }^{\dagger}$, Yingying Chen ${ }^{\sharp}$ \\ ${ }^{\dagger}$ WINLAB, Rutgers University, North Brunswick, NJ, USA \\ †\{shubhamj, gruteser\}@winlab.rutgers.edu \\ $\S$ Politecnico di Torino, Torino, Italy \\ scarlo.borgiattino@polito.it \\ \#Stevens Institute of Technology, Hoboken, NJ, USA \\ $\sharp\{$ yren2, yingying.chen\}@stevens.edu
}

\begin{abstract}
This paper studies the use of positioning techniques for sensing when pedestrians are at an increased risk of a traffic accident. Such sensing techniques could support augmented reality applications that increase pedestrian safety. We discuss requirements for pedestrian risk detection from rural to urban environments and consider algorithms relying on inertial and positioning sensors for distinguishing safe and unsafe walking locations. We study the limits of this approach through walking trials in different environments.
\end{abstract}

\section{Keywords}

Pedestrian Safety; Smartphone; Localization; Augmented Reality; GPS

\section{Categories and Subject Descriptors}

C.2.4 [Computer-Communication Networks]: Distributed SystemsDistributed Applications; H.5.m. [Information Interfaces and Presentation (e.g. HCI)]: Miscellaneous; C.3 [Special-Purpose and Application-based Systems]: Real-time and embedded systems

\section{INTRODUCTION}

As mobile and wearable computing technologies pose increasing distractions, an important role of augmented reality technology can be to steer our attention back to the real world. This role can be particularly valuable in a pedestrian safety context. Traffic accidents with pedestrians still account for a significant number of injuries or fatalities and there is mounting evidence that mobile device distractions of pedestrians are exacerbating this problem. From 2000 to 2009, the United States saw more than 47,700 pedestrians deaths in traffic accidents and 688,000 pedestrians were injured [11]. Pedestrian deaths account for nearly $14 \%$ of all traffic fatalities [18]. According to a study, 26\% of pedestrians text or email, $51 \%$ talk on the phone and $36 \%$ listen to music while cross-

Permission to make digital or hard copies of all or part of this work for personal or classroom use is granted without fee provided that copies are not made or distributed for profit or commercial advantage and that copies bear this notice and the full citation on the first page. Copyrights for components of this work owned by others than ACM must be honored. Abstracting with credit is permitted. To copy otherwise, or republish, to post on servers or to redistribute to lists, requires prior specific permission and/or a fee. Request permissions from permissions@acm.org.

MARS'14, June 16, 2014, Bretton Woods, New Hampshire, USA.

Copyright 2014 ACM 978-1-4503-2823-4/14/06 ...\$15.00.

http://dx.doi.org/10.1145/2609829.2609834. ing the street [15]. Distracted walking has also attracted significant media attention $[13,6]$. Mobile augmented reality technology could warn pedestrians when they are about to step into the street, using a variety of techniques. For example, they could underlay the composed text message of the user with a camera view of the street and a "Look Up!" notification. With a phone-to-vehicle communication system, it would also be possible to warn oncoming drivers.

Related Work. To support such applications and trigger notifications at the right time, mobile devices must sense and evaluate accident risks. Prior work in this area has focused on detection of oncoming cars using cell phone cameras as special sensing hardware. Gandhi et. al. [12] provide an overview of video, radar and laser distance measurement approaches for active pedestrian safety. RFID-based approach is discussed by Fackelmeier et. al. [8]. This approach doesn't need line of sight, but has a limited communication range and needs additional device to be carried by the pedestrian and the car. Another approach that needs no line of sight and is based on 3G and WLAN is presented by Sugimoto et. al. [20]. David et. al. [7, 9, 21] present a radio approach that assumes that the GPS location is precise up to 10 to $80 \mathrm{~cm}$. Their solution also relies on an external server, with a considerable connection establishment time, to coordinate communication between pedestrian and driver. They also add movement recognition to the radio-based solution [10]. Another pedestrian safety app by Wang et. al. [22] uses the smartphone's rear camera to detect vehicles approaching the pedestrian when he is talking and walking. This approach works only when the pedestrian is on a call and can easily drain the smartphone's computational resources and battery.

Approach. In this paper, we ask whether mobile devices can use their in-built sensing capabilities for pedestrian risk assessment. With the plethora of user interaction designs that augmented reality technology provides, we believe that the problem can be relaxed from detecting imminent collision to sensing increased pedestrian risk. Augmented reality interfaces offer many choices for subtler awareness cues rather than only raising startling alarms. Leaving aside the specific user interaction design, we focus on defining relevant pedestrian risk scenarios and studying whether mobile positioning and inertial sensing techniques can sense scenarios that pose increased risk. In summary, the contributions of this paper are:

- identifying pedestrian risk scenarios that are amenable to detection with in-built sensors

- defining requirements for a positioning and inertial sensing approach

- evaluate the limits of positioning techniques across these scenarios for pedestrian in-street detection 


\section{SCENARIOS AND CHALLENGES}

With a vehicle safety communication system such as the DSRC [5, 4] system supported by the US Department of Transportation, it is feasible for a pedestrian's device to notify oncoming vehicles when the pedestrian is in-street. The vehicle could alert the driver, perhaps using augmented reality displays on the windshield. The requirements and challenges for smartphone-based in-street detection techniques differ significantly across scenarios. In particular, in-street detection can assume different meanings depending on the environment. We discuss some of these scenarios here, and the corresponding application assumptions.

- Rural Out-of-Town Environments. In out-of-town environments such as rural roads or highways, pedestrians are rare and not expected by most drivers. Thus walking along such roads, which typically do not have sidewalks, can be particularly hazardous. Since pedestrians are sparse and tend to walk along roads for extended periods, the accuracy requirements on a positioning system are relatively low, in the order of tens of meters. It thus suffices to let an approaching driver know if a pedestrian is walking along a street.

- Suburban Environments. In suburban or residential areas, there may or may not be sidewalks, and one may expect occasional pedestrians, walking in the street (when no sidewalks present) or on the sidewalk. In the absence of sidewalk, any approaching car can be warned. For a pedestrian walking on the sidewalk, solely detecting that there is a pedestrian walking along the street would result in uselessly warning an approaching driver. It is thus beneficial to identify the events when this person may be stepping into the street, putting himself at a higher risk of being hit by car.

- Urban Environments. Most urban downtowns and cities are well developed and have sidewalks, with a large number of pedestrians walking along the street. Just knowing that a pedestrian is walking along a street would create far too many warnings for drivers and cause warning fatigue. In such a scenario, more fine-grained differentiation of pedestrians at risk is not only beneficial but necessary. Since a pedestrian is usually safe when walking on the sidewalk, our approach is to identify pedestrians that are in the roadway. Accurate detection of street crossing is therefore a key concern.

Considering the application scenarios listed here, it is evident that outdoor walking activity detection, determining that they occur near streets (street matching) and crossing detection are key building blocks. Those rely on localization, which is the biggest challenge for such a detection venture. A careful analysis of these scenarios also reveals that accurate positioning is not a stringent requirement in rural areas, but is increasingly valuable in suburban, and an absolute necessity in urban environments. While GPS localization might work well in some rural environments with no or very few buildings around, using GPS for street level localization in suburban and urban environments might face more significant difficulties. To better understand these challenges, we therefore study the limits of such a positioning-based approach in the latter two environments based on sample algorithms we describe next.

\section{DESIGN AND METHODOLOGY}

Our primary focus is to test the limits of position-based detection for the scenarios discussed in Section 2. Here we outline how position-based sensing could be part of a pedestrian risk assessment

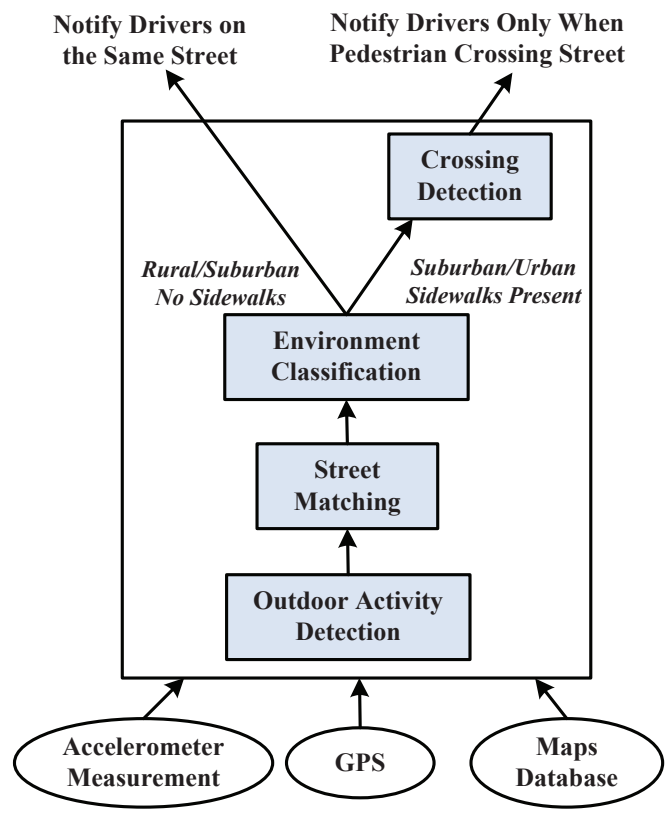

Figure 1: System overview.

system. Given that a large number of people use smartphones, we explore smartphone-based techniques to localize pedestrians and determine their position with respect to the street-sidewalk frame of reference.

Fig 1 provides a system overview. The bottom layer depicts the raw inputs available from the smartphone. This includes the GPS, accelerometer and maps data. The components in the box characterize the system. The accelerometer and GPS data help determine when a person is walking outdoor. Using the maps data and the GPS location, we can identify the street the pedestrian is walking along. Using more detailed information available from a maps database, we can classify the environment as rural, suburban or urban. We also identify whether or not the street has sidewalks. In case of a pedestrian walking along a street in a no sidewalks ru$\mathrm{ral} /$ suburban environment, the system can notify all the vehicles approaching the pedestrian. In the presence of sidewalks, the system runs an additional crossing detection algorithm, that identifies when a pedestrian is in-street (not on the sidewalk) and notifies only when such crossing events occur. The system components are discussed here in more detail.

Outdoor Activity Detection. Prior to running a detection on the pedestrian's location, we want to verify that this is done only when the pedestrian is outdoors. There exist multiple works on distinguishing whether the user is indoor or outdoor, based on GPS information, or accelerometer data tracking $[24,19,16]$. We can use these techniques to determine when a person is walking outdoor.

Street Matching. Once we have established that a person is walking outdoors, we use the location coordinates to determine whether the pedestrian is near a street by matching the location against a map. An approach similar to existing map matching techniques for vehicles [23, 14], can be used to identify the street a pedestrian is walking along. This technique identifies the street closest in distance from the pedestrian's current location. This would allow determining the vehicles driving on the same street as the pedestrian and enable targeted warnings of both drivers or pedestrians. In the context of a vehicle safety communication system, this information could be shared with vehicles over radio links 


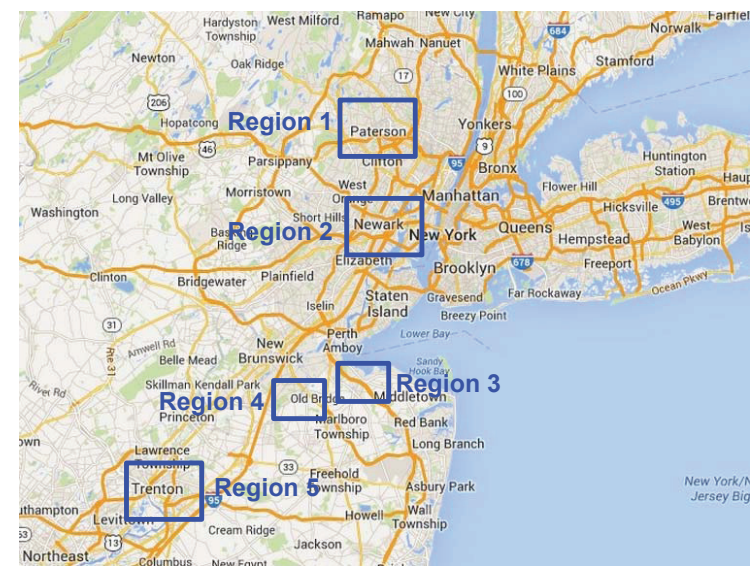

Figure 2: Regions for sidewalk presence heuristics validation.

such as $802.11 \mathrm{p}$ (which have been demonstrated in smartphones) with vehicles. When approaching intersection, two different streets appear to be at the same distance from the pedestrian. This is indeed useful, because at an intersection, cars traveling on both streets must be alerted if the pedestrian is in-street.

Environment Classification. The GPS location and a relevant maps database allows us to classify the area the pedestrian is walking in, as discussed in Section 2. It provides us an estimate of the detection requirement for the area. For a pedestrian in a rural environment where there are no sidewalks, we deduce that the pedestrian is walking in-street and that it is adequate to identify the street, and notify the cars driving on that street. In an urban location, it becomes imperative that the algorithm identifies potentially unsafe transitions into the street and alerts the cars only in relevant occurrences of such events. We can obtain the underlying street network information and check for the presence of sidewalks using OpenStreetMap [2].

OpenStreetmap is an open-source maps database that uses a topological data structure composed of nodes, ways, relations and tags. Tags are used to store metadata about the map objects [3]. Of the various road tags in the dataset, the sidewalk tag, location tag and highway tag, are used in our heuristics to determine if a street has sidewalk. These tags are able to show roads' information on sidewalk, location and road type, respectively. Using the sidewalk tag it is possible to indicate if a sidewalk is present on either both sides, the left side, or the right side of a street.

For validating our heuristics, we randomly select 30 streets from 5 regions in New Jersey. These regions are shown in Fig 2. For 6 streets in each region, we wanted to determine if it has a sidewalk or not. We acquire the information from OpenStreetMap and find the corresponding street on Google maps to compare our identification with the ground truth. If it is consistent, we say that the sidewalk is accurately detected. We calculate the sidewalk detection rate, which is the percentage of sidewalks that are accurately identified. We observe that we can achieve $94 \%$ sidewalk detection rate. This observation indicates that our heuristics can identify streets with sidewalks accurately. The coverage for the sidewalk tag is variable around the U.S., being best in Washington DC [1], Toronto and a number of other places.

Crossing Detection. As a final step, we need to detect when a pedestrian is in-street. It is fairly straightforward when there is no sidewalk present. We identify this as walking in street, potentially risky and warn any oncoming cars. In dense urban environments, with sidewalks and pedestrians abound, we do not want to send too frequent unnecessary alerts to pedestrians and approaching cars. Hence, more fine-grained information about pedestrians that are actually in the street is useful. This occurs usually when the person is crossing a street. Our algorithm achieves crossing detection by predicting a pedestrian's path of motion and checking if it intersects with any of the streets nearby. Such an intersection of the pedestrian's predicted path and the street centerline indicates when the user might be purposed to cross the street. To predict the path of motion, we extrapolate a user's heading by a distance $d$. In our scenario, the streets are two-way streets, single lane in each direction. The typical lane width in New Jersey is about 3.5 meters [17]. We chose $d$ accordingly.

\section{EXPERIMENT DESCRIPTION}

To test the proposition developed in the earlier section, we use the GPS information from the pedestrian's smartphone and analyze the extent to which positioning technologies can support in-street detection. The GPS on the smartphone provides us with useful information, such as the latitude and longitude for a pedestrians' location, their heading, speed and accuracy of the location provided.

We chalked out two separate test paths. 15 volunteers from our lab walked along these two test paths for the purposes of data collection. An Android application continuously collected sensor data. The test paths are shown in Fig 3. The path in Fig 3(a) is a suburban test path that includes a residential area, where most buildings have two floors. This area had a sidewalk only along one street, while for the rest of the path the pedestrian had to walk in the street, along the edge. The street was crossed for a total of 14 times on this path. The exact same path was traversed 20 times. This path incorporated various turning and street crossing scenarios.

Our second test path, as shown in Fig 3(b) was the downtown area of a small city, New Brunswick. Fig 3(b) shows only one of the many paths we covered. The street was crossed about 10-12 times in each trial. 12 different people walked a total of 28 similar loops for data collection. This test bed is a well developed urban area and had sidewalks throughout.

For both the test paths, the pedestrian carried the phone in hand or in pocket. The Android application in context recorded raw timestamped data from GPS, Accelerometer, Gyroscope, Magnetometer, Rotation Vector, Linear Acceleration and Gravity Acceleration. The information logged from the GPS was the latitude, longitude, bearing, speed, time of fix, accuracy and number of satellites used for that fix. We set the GPS to log data at the maximum available rate, which allows us to obtain one GPS location per second, on an average. The application also allows us to record the ground truth, i.e. the exact moment when the pedestrian enters a street, by touching a button on the screen. We use these timestamps to compare and evaluate the detection performance of our algorithm.

\section{EVALUATION}

Keeping in mind the gravity of pedestrian risk detection applications, we evaluate the performance of GPS based algorithms for such risk awareness. We also want to explore how this limit varies with environments. We selected suburban and urban scenarios because they are more challenging environments with a complex infrastructure. Rural out-of-town environments can be handled by existing localization techniques as discussed in Section 3. We also analyze detection delay, rather than just detection accuracy, since timeliness is important for the effectiveness of pedestrian safety warnings. 


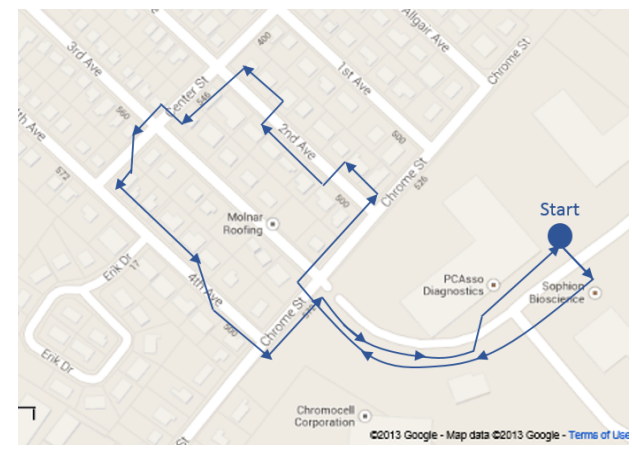

(a) Suburban test path.

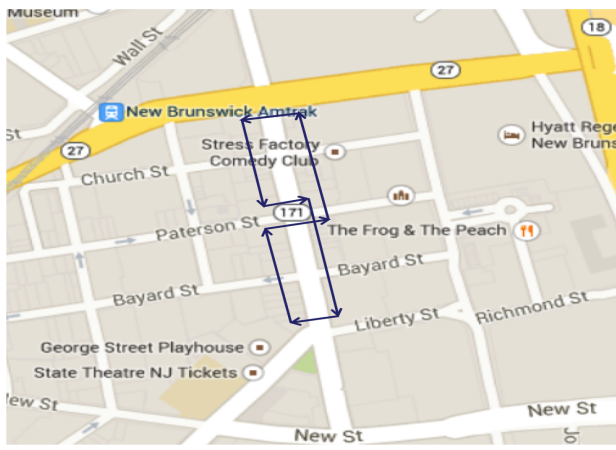

(b) New Brunswick test path.

Figure 3: Experiment test paths.

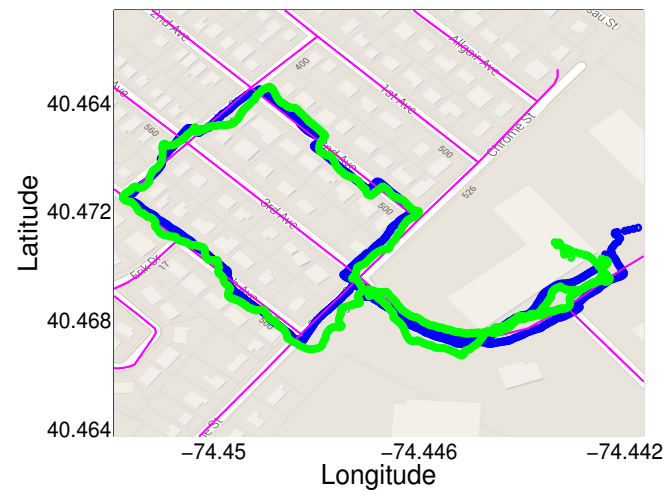

(a) GPS trace in suburban test bed with phone in hand (blue trace) and phone in pocket (green trace).

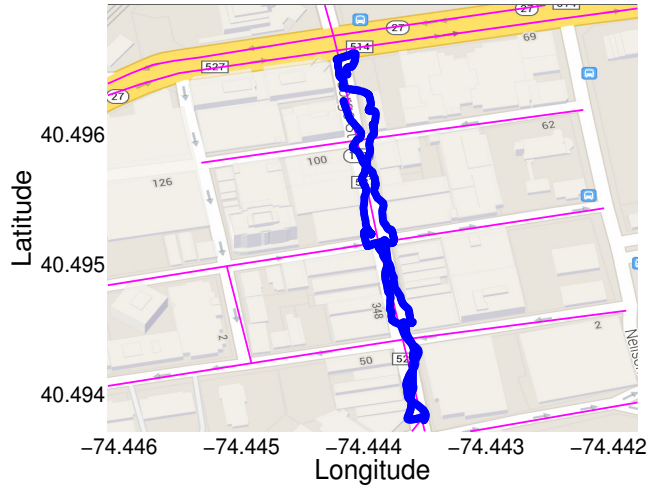

(b) GPS trace in urban test bed.

Figure 4: GPS traces plotted on underlying street network.

We first show here how the collected GPS traces look as compared to the actual path walked. The GPS trace from one of the trials for the suburban test bed is plotted on an underlying street map in Fig 4(a). The magenta lines are the center of the streets obtained from OpenStreetMap, the blue path is the GPS trace when the phone is in the user's hand, and the green path is the GPS trace when the phone is in user's pocket. It is evident from these traces that the GPS positioning accuracy deteriorates when the phone is in the pedestrian's pocket, compared to when in hand. Fig 4(b) shows the GPS trace of the urban downtown, plotted on the underlying street network. This trace substantiates that the GPS positioning quality declines rapidly in urban areas.

In-street detection for rural and suburban scenarios can be performed using the location provided by GPS and the sidewalk tag from OpenStreetMap. The heuristic used for this approach was discussed in the earlier section. For urban settings with sidewalks, we want to identify the events when a pedestrian enters the street. Therefore, we implement and evaluate a crossing detection algorithm based on GPS-alone.

Crossing Detection Performance Evaluation. Fig 5 shows GPS traces from segments of one of the trial walks. The magenta lines mark the center of the streets obtained from OpenStreetMap. The black dashed arrows indicate the actual path walked by the pedestrian and the blue path is the GPS trace obtained from the smartphone. The green lines are the predicted path of motion obtained by extending the pedestrian's GPS bearing by a distance $d$ (4 meters in this case), at each location update. Each time an intersection of the predicted path with the street is detected, a crossing prediction is said to be made. A red marker marks this crossing prediction. Based on whether or not the pedestrian crossed the street, this prediction can be a true detection or a false alarm.

Fig 5(a) shows two accurately detected crossings, identified by the intersection of the predicted path and the street. The red markers are the points where a predicted path intersects with the line marking the center of the street. An example false positive is shown in Fig 5(b). In this example, the pedestrian turned around the corner at an intersection (shown by the blue GPS trace) to walk along the perpendicular street, rather than crossing that street. This false alarm was caused by the change in the pedestrian's path of motion. GPS positioning suffers from delays in location updates and hence fails to capture the unanticipated changes in the pedestrian's path and bearing. A false negative is a missed detection, mostly caused due to GPS inaccuracy. Fig 5(c) is an example of a missed detection. In this case, the GPS is unsuccessful in accurately identifying which side of the street the pedestrian is on, resulting in missing the crossing.

From the discussion of the extrapolated path length $d$ in the previous section, we can see that the longer this extended distance, the earlier the crossing detection would be. On the other hand, a longer extended path would also lead to more false positives. To analyze how this path length affects our detection, we varied $d$ over a range of $0.1 \mathrm{~m}$ to $10 \mathrm{~m}$, in steps of $0.1 \mathrm{~m}$. 


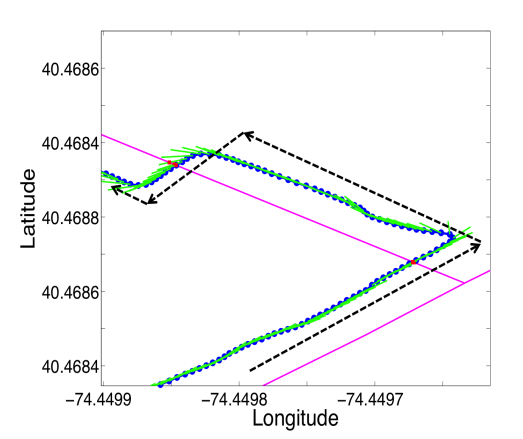

(a) Examples for correct crossing detections.

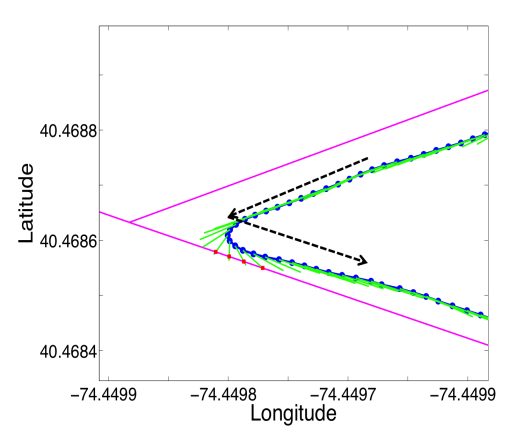

(b) An example false detection caused by change in bearing.

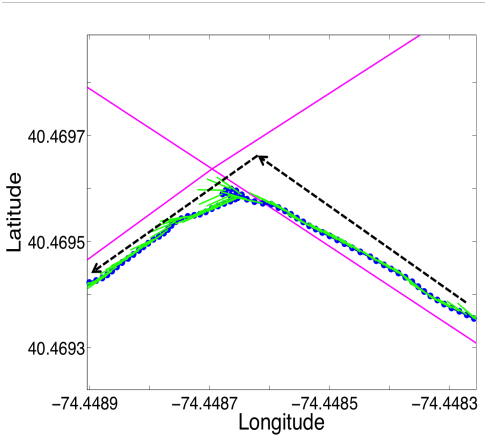

(c) An example missed detection caused by GPS inaccuracy.

Figure 5: Examples of various crossing detection scenarios. The magenta lines mark the center of the street. The black arrows indicate the actual path walked. The blue trace is the person's GPS trajectory. The green lines are the predicted path of motion. The red markers are the points where a predicted path intersects with the line marking the center of the street.

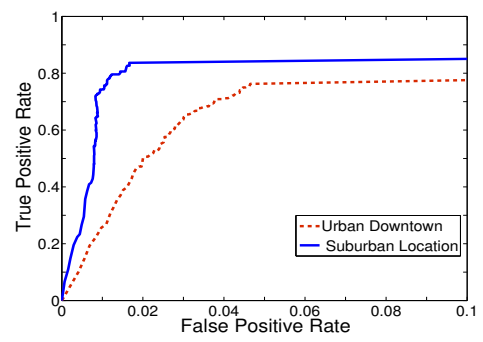

(a) In-street detection performance for urban and suburban testbeds at ground truth window $=5$ seconds.

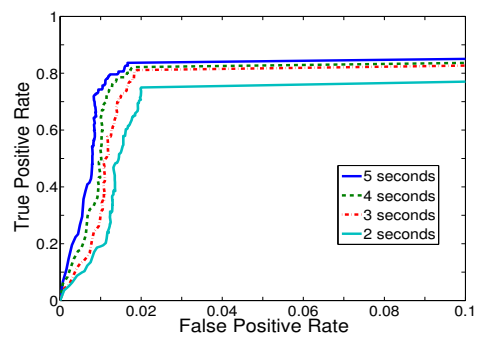

(b) In-street detection performance for the suburban test bed at varying ground truth windows.

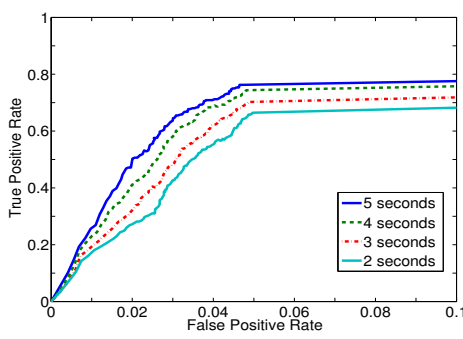

(c) In-street detection performance for the urban test bed at varying ground truth windows.

Figure 6: Crossing street detection performance analysis.

Fig 6(a) shows the Receiver Operating Characteristic (ROC) curves for the suburban and urban test beds. This curve plots the true positive rate against the false positive rate. We define a true positive as a detection made at most 8 seconds before and $k$ seconds after the exact instant the pedestrian entered the street. As discussed earlier, the entrance time was recorded by means of an Android application. In Fig 6(a), the value of $k$ used is 5 seconds, varied over $d$. This implies that for any detection to be counted as a true detection, the warning can occur as late as 5 seconds from the time of the exact entrance into the street.

We refer to this window as the ground truth window. For practically useful applications, the allowed time $k$, after the exact entrance must be small. This implies that the event must be detected as soon as the person enters the street, or within the allowable time window $k$. We vary $k$ to assess the algorithm for delay performance. Fig 6(b) shows the ROC curves for the suburban area for varying ground truth windows. We see that the algorithm provides a very good detection rate of $85 \%$ with as few as $1.8 \%$ false positives. This performance degrades as the allowed delay $(k)$ after the actual entrance is decreased.

Fig 6(c) shows the performance for the crossing detection algorithm in an urban environment, such as the downtown of a small city. We can observe a visible drop in the performance compared to that in a suburban environment, with a detection rate of $78 \%$ for $4.5 \%$ false positives. The maximum rate at which we can sample GPS is approximately once per second. It is evident from the per- formance curves that GPS does not work well for applications with a stringent timing and fine grained localization requirement, such as the pedestrian safety applications.

\section{DISCUSSION AND CONCLUSION}

We analyzed the performance of positioning and inertial sensing techniques for pedestrian in-street detection. We identified in-street detection in rural, out-of-town areas as a scenario that is likely to be feasible and our experiments show promising results even for sidewalk-street differentiation in suburban environments, but it still presents a challenge in urban environments. It is evident that GPS does not serve well the fine-grained positioning needs of pedestrian safety applications in dense urban environments. It is impaired with large errors in positioning and delays in detection, rendering it unfit for such critical applications.

One approach for further work that might overcome the many false positives in the GPS-only prediction algorithm, that occur due to GPS location and bearing inaccuracy, is the use of inertial sensors to detect key movement features by pedestrians. For example, we saw that GPS fails to capture the sudden changes in the pedestrian's path of motion close to an intersection. Such sudden turns can be detected with the in-built gyroscope in a smartphone. Fig 7 shows a plot of time vs gyroscope magnitude from a walking trial. Here, the vertical green lines depict the ground truth and mark the exact time instance when a user turned and the red markers mark 


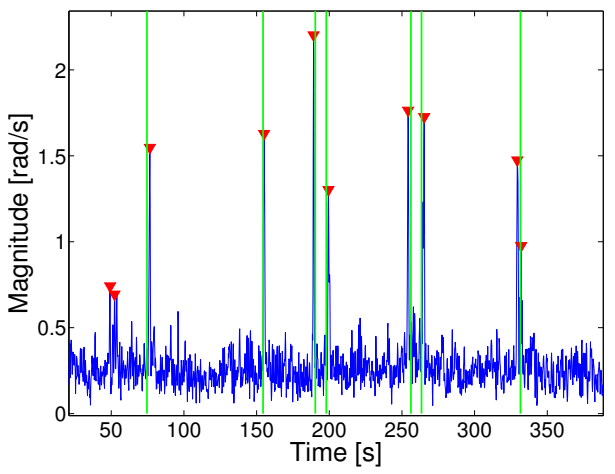

Figure 7: Turns detected by gyroscope.

the turn detected by our algorithm, based on gyroscope magnitude. We can see that most turns are detected.

Together such risk assessments can enable new applications that warn distracted pedestrians as well as drivers, when a safety communication system is available. As our electronic devices are increasingly drawing our attention away from real world hazards, we believe that this will be an important feature of future mobile augmented reality systems.

\section{ACKNOWLEDGEMENTS}

We are thankful to all the participants who walked for collecting the dataset used for evaluation in this paper. This material is based in part upon work supported by Qualcomm and the National Science Foundation under Grant No. CNS-1329939, CNS-1217387 and CCF-1018270.

\section{REFERENCES}

[1] ito world. http://goo.gl/sWMVHT.

[2] Openstreetmap. http: //www.openstreetmap.org/.

[3] Openstreetmap wiki. http://goo.gl/66Vlki.

[4] U.S. Department of Transportation, DSRC. http: //www.its.dot.gov/factsheets/dsrc_factsheet.htm.

[5] OKI Electric Industry Co. DSRC attachment for mobile phones, January 2009.

[6] UK Daily Mail. Texting while walking blamed in the nationwide increase of pedestrian deaths. http://goo.gl/kakggc.

[7] K. David and A. Flach. Car-2-x and pedestrian safety. Vehicular Technology Magazine, 2010.

[8] Andreas Fackelmeier, Christian Morhart, and Erwin Biebl. Dual frequency methods for identifying hidden targets in road traffic. In Advanced Microsystems for Automotive Applications. 2008.
[9] A. Flach and K. David. Combining radio transmission with filters for pedestrian safety: Experiments and simulations. In Vehicular Technology Conference Fall, 2010.

[10] A. Flach, A.Q. Memon, Sian Lun Lau, and K. David. Pedestrian movement recognition for radio based collision avoidance: A performance analysis. In Vehicular Technology Conference (VTC Spring), 2011.

[11] Transportation for America. Dangerous by design, 2011.

[12] T. Gandhi and M.M. Trivedi. Pedestrian protection systems: Issues, survey, and challenges. Intelligent Transportation Systems, IEEE Transactions on, 2007.

[13] Boston Globe. Put that phone down and just walk. http: //goo.gl/EwgrMg.

[14] Joshua S Greenfeld. Matching gps observations to locations on a digital map. In National Research Council (US). Transportation Research Board. Meeting (81st: 2002: Washington, DC). Preprint CD-ROM, 2002.

[15] Liberty Mutual Insurance. Study shows three out of five pedestrians prioritize smartphones over safety when crossing street, June 2013.

[16] D. H. Kim, Y. Kim, D. Estrin, and M. B. Srivastava. Sensloc: sensing everyday places and paths using less energy. In Embedded Networked Sensor Systems (SenSys), 2010.

[17] New Jersey Department of Transportation. Roadway design manual.

[18] U.S Department of Transportation. Traffic safety facts, August 2013.

[19] C. Qin, X. Bao, R. Roy Choudhury, and S. Nelakuditi. Tagsense: a smartphone-based approach to automatic image tagging. In International Conference on Mobile Systems, Applications, and Services (MobiSys), 2011.

[20] C. Sugimoto, Y. Nakamura, and T. Hashimoto. Prototype of pedestrian-to-vehicle communication system for the prevention of pedestrian accidents using both $3 \mathrm{~g}$ wireless and wlan communication. In $3 r d$ International Symposium on Wireless Pervasive Computing, 2008.

[21] C. Voigtmann, Sian Lun Lau, and K. David. Evaluation of a collaborative-based filter technique to proactively detect pedestrians at risk. In Vehicular Technology Conference (VTC Fall), 2012.

[22] Tianyu Wang, Giuseppe Cardone, Antonio Corradi, Lorenzo Torresani, and Andrew T. Campbell. Walksafe: a pedestrian safety app for mobile phone users who walk and talk while crossing roads. In Proceedings of the Twelfth Workshop on Mobile Computing Systems \& Applications, HotMobile '12.

[23] Christopher E White, David Bernstein, and Alain L Kornhauser. Some map matching algorithms for personal navigation assistants. Transportation Research Part C: Emerging Technologies, 2000.

[24] P. Zhou, Y. Zheng, Z. Li, M. Li, and G. Shen. Iodetector: A generic service for indoor outdoor detection. In Embedded Networked Sensor Systems (SenSys), 2012. 